\title{
Physiological Response of Flag Leaf Nutrition in Rice (Oryza sativa L.)
}

\author{
P. Shalini Pillai* and M. S. Surya \\ Department of Agronomy, Kerala Agricultural University, College of Agriculture, Vellayani, \\ Thiruvananthapuram, Kerala, India \\ *Corresponding author
}

\section{A B S T R A C T}

\section{Keywords}

Flag leaf nutrition, Rice, 19: 19: 19 complex, Potassium nitrate

\section{Article Info}

Accepted:

15 January 2021

Available Online:

10 February 2021
A field experiment was carried out during Kharif season at the Instructional Farm, College of Agriculture, Vellayani, Thiruvananthapuram, Kerala for assessing the effect of flag leaf nutrition (FLN) on the physiological response of rice, with PTB 52 (Aiswarya) as the test variety. Leaf area duration was observed to be longest with FLN of calcium nitrate @ 0.5 per cent and was at par with all nutrient sources except potassium nitrate. Spraying a combination of calcium nitrate, magnesium sulphate and 19:19:19 complex @ 0.5 per cent $\left(\mathrm{S}_{5}\right)$ recorded the highest specific leaf weight at flowering $\left(2.11 \mathrm{~g} \mathrm{~m}^{-2}\right)$ and foliar spraying of 19:19:19 complex @ 0.5 per cent $\left(2.58 \mathrm{~g} \mathrm{~m}^{-2}\right)$ recorded the highest value at harvest stage. Flag leaf nutrition with potassium nitrate at booting stage $\left(\mathrm{s}_{1} \mathrm{~g}_{1}\right)$ was significantly superior in terms of specific leaf weight $\left(2.57 \mathrm{~g} \mathrm{~m}^{-2}\right)$, relative growth rate and net assimilation rate. Highest chlorophyll was with 19:19:19 complex and carotenoid with potassium nitrate @ 0.5 per cent. Magnesium sulphate @ 0.5 per cent concentration $\left(\mathrm{S}_{3}\right)$ recorded the maximum total soluble protein $\left(1.04 \mathrm{mg} \mathrm{g}^{-1}\right)$ at booting stage and was at par with $S_{2}$ (calcium nitrate) and $S_{4}(19: 19: 19$ complex). The study revealed that KAU POP recommendation for high yielding medium duration wetland rice (FYM @ $5 \mathrm{t} \mathrm{ha}^{-1}+$ 90:45:45 $\mathrm{kg} \mathrm{NPK} \mathrm{ha}^{-1}$ ) supplemented with flag leaf nutrition of potassium nitrate or 19: 19: 19 complex @ 0.5 per cent concentration, 5 days prior to booting and 50 per cent flowering stages elicited significantly superior physiological response in lowland rice.

\section{Introduction}

Rice production is an integral part of the national economy, directly feeding more people than any other crop. The importance of rice is highlighted by the fact that it is the only crop to have acquired two years designated as "international" in its honour. The yield of rice under lowland conditions are almost always lower than that under irrigated or otherwise optimum conditions (Mackill et al., 1996). The low grain filling rate and grain weight implicating a reduced grain yield often result from limited carbohydrate supply (Yang et al., 2003). Yield is the culmination of a series of physiological and biochemical processes (Ashraf et al., 1994). Photosynthesis is the primary source of carbohydrates for grain filling, which has to be sustained by current photosynthesis of the topmost leaves (Tambussi et al., 2007), especially the flag leaf.. Flag leaf which 
subtends the emerging panicle is the premier source of photosynthetic energy in the reproductive phase of rice. In rice, 60 to 90 per cent of the total carbohydrates in the panicles at harvest are derived from the photosynthesis after panicle initiation (Tari et al., 2009). Leaf senescence during the reproductive and maturity stages is directly related to biomass production and grain yield of rice (Misra et al., 1997). Attempts, including nutrient management could be made for delaying the flag leaf senescence and this closely relates to the leaf physiology and metabolism. Identification of growth and physiological indices in analysis of factors affecting yield and its components assumes paramount importance since its stability determines the dry matter production which is a criterion of yield components. The present study was undertaken to assess the effect of flag leaf nutrition on the physiology of lowland rice.

\section{Materials and Methods}

A field experiment was conducted at the Instructional farm, College of Agriculture, Vellayani, Thiruvananthapuram, Kerala, India, during the first crop season (June October). The soil of the experimental site was sandy clay loam, acidic in reaction $(\mathrm{pH}-$ $5.50)$, high in organic carbon (1.78\%), medium in available nitrogen $(300.64 \mathrm{~kg}$ ha $\left.{ }^{1}\right)$, high in available phosphorus $(27.52 \mathrm{~kg} \mathrm{ha}$ $\left.{ }^{1}\right)$, medium in available potassium $(186.35 \mathrm{~kg}$ $\mathrm{ha}^{-1}$ ) and deficient in exchangeable calcium (117.85 kg ha ${ }^{-1}$ ), magnesium (113.72 kg ha $\left.{ }^{-1}\right)$ and available sulphur $\left(9.92 \mathrm{~kg} \mathrm{ha}^{-1}\right)$ The rice variety chosen for the study was Aiswarya (PTB 52). Sixteen treatment combinations i.e., $[(5 \times 3)+1]$ was laid out in randomized block design with three replications. The treatments were 0.5 per cent each of $S_{1}$ : potassium nitrate, $S_{2}$ : calcium nitrate, $S_{3}$ : magnesium sulphate, $S_{4}$ : 19: 19: 19 complex and $S_{5:}$ a combination $S_{2}+S_{3}+S_{4}$, sprayed at three crop growth stages viz., $\mathrm{G}_{1}$ : Booting stage, $\mathrm{G}_{2}$ : Booting + Flowering stages and $\mathrm{G}_{3}$ : Booting + Flowering + Milk stages, as compared against a control. The control was the Kerala Agricultural University package of practices (KAU POP) recommendation for medium duration rice - FYM @ $5 \mathrm{t} \mathrm{ha}^{-1}$, 90:45:45 NPK kg ha-1 . Flag leaf nutrition was given to supplement the control. Flag leaf nutrition was done at 5 days prior to booting (45 DAT), 5 days prior to 50 per cent flowering (60 DAT) and at milk stages (75 DAT) as per treatments, following the procedure of Fageria et al., (2009). Booting, flowering and milk stages were characterized as described by De Datta (1981). The nutrient sources $(0.5 \%)$ were sprayed along with an adjuvant ( $1 \mathrm{~mL}$ per $10 \mathrm{~L}$ of spray fluid) at a spray volume of $500 \mathrm{~L} \mathrm{ha}^{-1}$. Spraying was done after $3.00 \mathrm{pm}$ under calm atmospheric conditions. The individual plots were separated using screens to avoid the effect of any possible spray drift.

Flag leaf area at booting, flowering, harvest stages was calculated by leaf product method. The factor $(\mathrm{k})$ used were 0.75 (booting and flowering stages) and 0.67 (harvest stage).

Leaf area $\left(\mathrm{cm}^{2}\right)=$ Length $(\mathrm{cm}) \times$ Maximum width $(\mathrm{cm}) \mathrm{x} \mathrm{k}$

Leaf area duration (LAD) at booting, flowering and harvest stages was calculated using the formula suggested by Watson (1947) and expressed in days.

$L A D=\underline{L_{i}+\left(L_{i}+1\right) \times\left(t_{2}-t_{1}\right)}$

$\mathrm{L}_{\mathrm{i}}=\mathrm{LAI}$ at first stage; $\mathrm{L}_{\mathrm{i}}+1=\mathrm{LAI}$ at second stage

$\left(t_{2}-t_{1}\right)=$ time interval between stages in days

Specific leaf weight (SLW) was calculated by using the formula suggested by Pearce et al., (1968) and expressed in $\mathrm{g} \mathrm{m}^{-2}$. 


\section{SLW $=$ Leaf dry weight per plant $(\mathrm{g})$ \\ Leaf area per plant $\left(\mathrm{cm}^{2}\right)$}

Relative growth rate (RGR) at booting, flowering, and harvest stages was determined using the formulae of Williams (1946) and expressed in $\mathrm{mg} \mathrm{g}^{-1} \mathrm{day}^{-1}$.

$$
\mathrm{RGR}=\frac{\log _{\mathrm{e}} \mathrm{W}_{2}-\log _{\mathrm{e}} \mathrm{W}_{1}}{\mathrm{t}_{2}-\mathrm{t}_{1}}
$$

$\mathrm{W}_{1}$ and $\mathrm{W}_{2} \quad=$ plant dry weight $(\mathrm{g})$ at time $\mathrm{t}_{1}$ and $\mathrm{t}_{2}$ respectively

$\mathrm{t}_{2}-\mathrm{t}_{1} \quad=$ time interval in days

The method proposed by Williams (1946) was used for calculating the net assimilation rate (NAR) on leaf dry weight basis and the values were expressed as $\mathrm{mg} \mathrm{cm}^{-2} \mathrm{day}^{-1}$. Net assimilation rate was recorded at booting, flowering and harvest stages.

$$
\mathrm{NAR}=\frac{\mathrm{W}_{2}-\mathrm{W}_{1}}{\mathrm{t}_{2}-\mathrm{t}_{1}} \times \frac{\log _{\mathrm{e}} \mathrm{L}_{2}-\log _{\mathrm{e}} \mathrm{L}_{1}}{\mathrm{~L}_{2}-\mathrm{L}_{1}}
$$

$\mathrm{W}_{1}$ and $\mathrm{W}_{2} \quad=$ leaf dry weight $(\mathrm{mg})$ at $\mathrm{t}_{1}$ and $\mathrm{t}_{2}$ respectively

$\mathrm{L}_{1}$ and $\mathrm{L}_{2}=$ leaf area $\left(\mathrm{cm}^{2}\right)$ at $\mathrm{t}_{1}$ and $\mathrm{t}_{2}$ respectively

$\mathrm{t}_{2}-\mathrm{t}_{1}=$ time interval in days

Chlorophyll and carotenoid pigments of flag leaf and sheath were analyzed at booting, flowering and harvest stages, using the DMSO (Di methyl sulphoxide) method suggested by (Yoshida et al., 1976) and expressed in $\mathrm{mg} \mathrm{g}^{-1}$.

Total soluble protein of flag leaf was estimated at booting, flowering and harvest stages was estimated using simple protein-dry binding method of Bradford (1976) using bovine serum albumin as the standard and expressed as $\mathrm{mg} \mathrm{g}^{-1}$ fresh weight.

\section{Results and Discussion}

\section{Flag leaf area}

The flag leaf area varied significantly with the different nutrient sources at booting and flowering stages (Fig. 1). Flag leaf area was significantly higher under foliar feeding with potassium nitrate $\left(\mathrm{S}_{1}\right)$ at booting $\left(4.98 \mathrm{~cm}^{2}\right)$ and flowering $\left(8.11 \mathrm{~cm}^{2}\right)$ stages. While the effect of potassium nitrate was on par with that of $\mathrm{S}_{4}$ (19:19:19 complex) at booting stage, it remained at par with 19:19:19 complex $\left(S_{4}\right)$ and calcium nitrate $\left(S_{2}\right)$ at the flowering stage. Flag leaf area at harvest stage remained unaffected by the different nutrient sources. The interaction effect between nutrient sources and crop growth stages was observed to be significant at flowering and harvest stages. Foliar spraying of potassium nitrate @ 0.5 per cent at booting and flowering stages $\left(\mathrm{s}_{1} \mathrm{~g}_{2}\right)$ recorded the highest flag leaf area $\left(9.08 \mathrm{~cm}^{2}\right)$ at flowering stage and was at par with calcium nitrate at the same concentration at booting stage $\left(\mathrm{s}_{2} \mathrm{~g}_{1}\right)$. Foliar spray of calcium nitrate at booting, flowering and milk stages $\left(\mathrm{s}_{2} \mathrm{~g}_{3}\right)$ resulted in the highest flag leaf area $\left(11.65 \mathrm{~cm}^{2}\right)$ at harvest stage. Significant difference was observed in the flag leaf area between the treatments and the control at flowering stage. The treatment effect was significantly superior $\left(7.52 \mathrm{~cm}^{2}\right)$ to control $\left(6.38 \mathrm{~cm}^{2}\right)$ at this stage. Both potassium nitrate and calcium nitrate are good sources of nitrogen. Further foliar feeding of these sources might have resulted in better absorption and assimilation of nitrogen, resulting in higher flag leaf area.

\section{Leaf area duration}

Leaf area duration was observed to be longest (41.88 days) when KAU POP was supplemented with FLN of calcium nitrate @ 
0.5 per cent and was at par with all the other nutrient sources except potassium nitrate (Fig. 2). Leaf area duration remained unaffected by crop growth stages and their interaction. Prevalence of $\mathrm{Ca}^{2+}$ cation plays a key role in cellular functions and enzyme activity (Bush,
1995). The presence of $\mathrm{Ca}^{2+}$ can also result in more rational utilization of soil nitrogen and more active assimilation of $\mathrm{NO}_{3}^{-} \mathrm{N}$ in roots and leaves (Kondratev et al., 1984), leading to delay in senescence as evidenced by better leaf area duration.

Table.1 Effect of nutrient sources, growth stages and their interaction on specific leaf weight $\left(\mathrm{g} \mathrm{m}^{-2}\right)$, relative growth rate $\left(\mathrm{mg} \mathrm{g}^{-1} \mathrm{day}^{-1}\right)$ and net assimilation rate $\left(\mathrm{mg} \mathrm{cm}^{-2}\right.$ day $\left.^{-1}\right)$

\begin{tabular}{|c|c|c|c|c|c|c|c|c|c|}
\hline \multirow[t]{2}{*}{ Treatments } & \multicolumn{3}{|c|}{ Booting } & \multicolumn{3}{|c|}{ Flowering } & \multicolumn{3}{|c|}{ Harvest } \\
\hline & SLW & RGR & NAR & SLW & RGR & NAR & SLW & RGR & NAR \\
\hline \multicolumn{10}{|l|}{ Nutrient sources } \\
\hline$S_{1}$ & 1.31 & 0.174 & 3.76 & 2.09 & 0.057 & 2.23 & 2.54 & 0.028 & 0.71 \\
\hline $\mathbf{S}_{2}$ & 1.37 & 0.078 & 1.95 & 1.64 & 0.047 & 2.23 & 2.30 & 0.019 & 0.26 \\
\hline $\mathbf{S}_{3}$ & 1.10 & 0.074 & 2.32 & 2.09 & 0.047 & 2.44 & 2.49 & 0.013 & 0.56 \\
\hline $\mathbf{S}_{4}$ & 1.22 & 0.087 & 2.57 & 2.07 & 0.052 & 2.58 & 2.58 & 0.027 & 0.44 \\
\hline $\mathbf{S}_{5}$ & 1.12 & 0.076 & 2.10 & 2.11 & 0.046 & 2.38 & 2.18 & 0.021 & 0.48 \\
\hline \multicolumn{10}{|l|}{ Growth stages } \\
\hline $\mathrm{G}_{1}$ & 1.29 & 0.135 & 2.66 & 2.03 & 0.060 & 2.37 & 2.50 & 0.024 & 0.46 \\
\hline $\mathbf{G}_{2}$ & 1.21 & 0.080 & 2.70 & 2.04 & 0.051 & 2.39 & 2.37 & 0.019 & 0.41 \\
\hline $\mathbf{G}_{3}$ & 1.16 & 0.079 & 2.26 & 1.93 & 0.059 & 2.36 & 2.39 & 0.021 & 0.59 \\
\hline \multicolumn{10}{|l|}{ Interaction effects } \\
\hline $\mathrm{s}_{1} \mathrm{~g}_{1}$ & 1.60 & 0.358 & 4.19 & 2.57 & 0.049 & 2.13 & 2.75 & 0.027 & 0.85 \\
\hline $\mathbf{s}_{1} \mathbf{g}_{2}$ & 0.99 & 0.083 & 3.80 & 2.03 & 0.048 & 2.33 & 2.16 & 0.029 & 0.73 \\
\hline $\mathbf{s}_{1} \mathbf{g}_{3}$ & 1.34 & 0.080 & 3.30 & 1.66 & 0.043 & 2.25 & 2.71 & 0.027 & 0.55 \\
\hline $\mathbf{s}_{2} \mathbf{g}_{1}$ & 1.49 & 0.078 & 1.96 & 1.59 & 0.049 & 2.16 & 2.29 & 0.020 & 0.22 \\
\hline $\mathbf{S}_{2} \mathbf{g}_{2}$ & 1.33 & 0.080 & 2.13 & 1.64 & 0.048 & 2.28 & 2.27 & 0.019 & 0.42 \\
\hline $\mathbf{s}_{2} \mathbf{g}_{3}$ & 1.29 & 0.077 & 1.75 & 1.70 & 0.043 & 2.26 & 2.36 & 0.019 & 0.14 \\
\hline $\mathbf{s}_{3} g_{1}$ & 1.00 & 0.072 & 2.26 & 1.90 & 0.049 & 2.54 & 2.33 & 0.013 & 0.46 \\
\hline $\mathbf{s}_{3} \mathbf{g}_{2}$ & 1.27 & 0.074 & 2.29 & 2.18 & 0.050 & 2.26 & 2.59 & 0.012 & 0.35 \\
\hline $\mathbf{s}_{3} g_{3}$ & 1.02 & 0.078 & 2.42 & 2.19 & 0.042 & 2.51 & 2.55 & 0.014 & 0.87 \\
\hline $\mathbf{s}_{4} \mathbf{g}_{1}$ & 1.14 & 0.089 & 2.48 & 2.06 & 0.047 & 2.65 & 2.81 & 0.027 & 0.45 \\
\hline $\mathbf{s}_{4} \mathbf{g}_{2}$ & 1.27 & 0.086 & 3.38 & 2.16 & 0.053 & 2.55 & 2.66 & 0.026 & 0.21 \\
\hline $\mathbf{S}_{4} \mathbf{g}_{3}$ & 1.25 & 0.085 & 1.87 & 1.99 & 0.058 & 2.56 & 2.29 & 0.027 & 0.65 \\
\hline $\mathbf{S}_{5} \mathbf{g}_{1}$ & 1.22 & 0.078 & 2.40 & 2.03 & 0.047 & 2.37 & 2.32 & 0.034 & 0.35 \\
\hline $\mathbf{S}_{5} \mathbf{g}_{2}$ & 1.21 & 0.077 & 1.94 & 2.17 & 0.048 & 2.52 & 2.17 & 0.010 & 0.34 \\
\hline $\mathrm{S}_{5} \mathrm{~g}_{3}$ & 0.92 & 0.074 & 1.96 & 2.14 & 0.043 & 2.26 & 2.03 & 0.018 & 0.75 \\
\hline Treatment mean & 1.22 & 0.090 & 2.5 & 2.00 & 0.050 & 2.37 & 2.41 & 0.020 & 0.48 \\
\hline Control & 1.29 & 0.076 & 2.04 & 2.11 & 0.040 & 2.15 & 2.14 & 0.012 & 0.35 \\
\hline $\operatorname{SEm}( \pm): S$ & 0.13 & 0.040 & 0.23 & 0.08 & 0.001 & 0.08 & 0.10 & 0.003 & 0.12 \\
\hline G & 0.10 & 0.031 & 0.18 & 0.06 & 0.001 & 0.06 & 0.08 & 0.002 & 0.09 \\
\hline SG & 0.23 & 0.067 & 0.38 & 0.15 & 0.004 & 0.16 & 0.18 & 0.005 & 0.20 \\
\hline $\mathrm{CD}(0.05): \mathrm{S}$ & NS & NS & 0.658 & 0.211 & 0.005 & 0.240 & 0.300 & 0.010 & NS \\
\hline G & NS & NS & NS & NS & NS & NS & NS & NS- & NS \\
\hline SG & NS & NS & NS & 0.435 & NS & NS & NS & NS & NS \\
\hline $\begin{array}{l}\text { Treatment } V s \\
\text { Control }\end{array}$ & NS & NS & NS & NS & NS & NS & NS & NS & NS \\
\hline
\end{tabular}


Table.2 Effect of nutrient sources, growth stages and their interaction on chlorophyll content in flag leaf, $\mathrm{mg} \mathrm{g}^{-1}$

\begin{tabular}{|c|c|c|c|c|c|c|}
\hline \multirow[t]{2}{*}{ Treatments } & \multicolumn{2}{|c|}{ Booting } & \multicolumn{2}{|c|}{ Flowering } & \multicolumn{2}{|c|}{ Harvest } \\
\hline & $\begin{array}{c}\text { Leaf } \\
\text { sheath }\end{array}$ & $\begin{array}{l}\text { Leaf } \\
\text { blade }\end{array}$ & $\begin{array}{l}\text { Leaf } \\
\text { sheath }\end{array}$ & $\begin{array}{l}\text { Leaf } \\
\text { blade }\end{array}$ & $\begin{array}{c}\text { Leaf } \\
\text { sheath }\end{array}$ & $\begin{array}{r}\text { Leaf } \\
\text { blade }\end{array}$ \\
\hline $\mathbf{S}_{1}$ & 0.41 & 0.68 & 0.58 & 2.23 & 0.40 & 0.62 \\
\hline $\mathbf{S}_{2}$ & 0.48 & 0.79 & 0.51 & 2.23 & 0.38 & 0.74 \\
\hline $\mathbf{S}_{\mathbf{3}}$ & 0.54 & 0.75 & 0.49 & 2.44 & 0.40 & 0.61 \\
\hline$S_{4}$ & 0.41 & 0.89 & 0.55 & 2.58 & 0.39 & 0.53 \\
\hline$S_{5}$ & 0.42 & 0.83 & 0.63 & 2.38 & 0.41 & 0.66 \\
\hline $\mathbf{G}_{1}$ & 0.49 & 0.67 & 0.55 & 2.37 & 0.40 & 0.58 \\
\hline $\mathbf{G}_{2}$ & 0.49 & 0.79 & 0.55 & 2.39 & 0.42 & 0.66 \\
\hline $\mathbf{G}_{3}$ & 0.39 & 0.91 & 0.56 & 2.36 & 0.37 & 0.65 \\
\hline $\mathbf{s}_{1} \mathbf{g}_{1}$ & 0.33 & 0.62 & 0.52 & 2.13 & 0.35 & 0.62 \\
\hline$s_{1} g_{2}$ & 0.45 & 0.71 & 0.46 & 2.33 & 0.42 & 0.62 \\
\hline $\mathbf{s}_{1} \mathbf{g}_{3}$ & 0.46 & 0.71 & 0.68 & 2.25 & 0.41 & 0.61 \\
\hline $\mathbf{s}_{2} \mathbf{g}_{1}$ & 0.61 & 0.73 & 0.54 & 2.16 & 0.38 & 0.61 \\
\hline $\mathbf{s}_{2} \mathbf{g}_{2}$ & 0.58 & 0.63 & 0.61 & 2.28 & 0.41 & 0.73 \\
\hline $\mathbf{s}_{2} \mathbf{g}_{3}$ & 0.45 & 1.02 & 0.58 & 2.26 & 0.45 & 0.89 \\
\hline $\mathbf{s}_{3} g_{1}$ & 0.57 & 0.65 & 0.54 & 2.54 & 0.38 & 0.69 \\
\hline$s_{3} g_{2}$ & 0.54 & 0.72 & 0.60 & 2.26 & 0.38 & 0.57 \\
\hline $\mathbf{s}_{3} g_{3}$ & 0.34 & 0.87 & 0.39 & 2.51 & 0.39 & 0.57 \\
\hline $\mathbf{s}_{4} \mathbf{g}_{1}$ & 0.43 & 0.62 & 0.64 & 2.65 & 0.34 & 0.51 \\
\hline $\mathbf{S}_{4} \mathbf{g}_{2}$ & 0.48 & 1.00 & 0.35 & 2.55 & 0.45 & 0.45 \\
\hline$s_{4} g_{3}$ & 0.37 & 1.05 & 0.50 & 2.56 & 0.41 & 0.63 \\
\hline $\mathbf{s}_{5} \mathbf{g}_{1}$ & 0.53 & 0.75 & 0.55 & 2.37 & 0.40 & 0.47 \\
\hline $\mathrm{s}_{5} \mathrm{~g}_{2}$ & 0.39 & 0.87 & 0.73 & 2.52 & 0.45 & 0.96 \\
\hline $\mathrm{s}_{5} \mathrm{~g}_{3}$ & 0.33 & 0.89 & 0.62 & 2.26 & 0.36 & 0.56 \\
\hline Treatment mean & 0.45 & 0.78 & 0.55 & 2.37 & 0.39 & 0.67 \\
\hline Control & 0.53 & 0.55 & 0.60 & 2.15 & 0.51 & 0.65 \\
\hline $\operatorname{SEm}( \pm): S$ & 0.034 & 0.075 & 0.033 & 0.082 & 0.029 & 0.050 \\
\hline G & 0.026 & 0.058 & 0.025 & 0.064 & 0.022 & 0.039 \\
\hline SG & 0.057 & 0.134 & 0.064 & 0.158 & 0.065 & 0.101 \\
\hline CD (0.05) : S & NS & NS & 0.096 & 0.240 & NS & NS \\
\hline G & NS & NS & NS & NS & NS & NS \\
\hline SG & NS & NS & NS & NS & NS & 0.291 \\
\hline Treatment Vs Control & NS & NS & NS & NS & NS & NS \\
\hline
\end{tabular}


Table.3 Effect of nutrient sources, growth stages and their interaction on carotenoid content in flag leaf, $\mathrm{mg} \mathrm{g}^{-1}$

\begin{tabular}{|c|c|c|c|c|c|c|}
\hline \multirow[t]{2}{*}{ Treatments } & \multicolumn{2}{|c|}{ Booting } & \multicolumn{2}{|c|}{ Flowering } & \multicolumn{2}{|c|}{ Harvest } \\
\hline & $\begin{array}{c}\text { Leaf } \\
\text { sheath }\end{array}$ & $\begin{array}{l}\text { Leaf } \\
\text { blade }\end{array}$ & $\begin{array}{c}\text { Leaf } \\
\text { sheath }\end{array}$ & $\begin{array}{l}\text { Leaf } \\
\text { blade }\end{array}$ & $\begin{array}{c}\text { Leaf } \\
\text { sheath }\end{array}$ & $\begin{array}{l}\text { Leaf } \\
\text { blade }\end{array}$ \\
\hline$S_{1}$ & 0.41 & 0.40 & 0.55 & 1.83 & 0.39 & 0.46 \\
\hline $\mathbf{S}_{2}$ & 0.54 & 0.47 & 0.58 & 1.17 & 0.41 & 0.46 \\
\hline $\mathbf{S}_{3}$ & 0.48 & 0.52 & 0.51 & 1.34 & 0.38 & 0.43 \\
\hline $\mathbf{S}_{4}$ & 0.42 & 0.60 & 0.49 & 1.44 & 0.40 & 0.60 \\
\hline $\mathbf{S}_{5}$ & 0.41 & 0.59 & 0.63 & 1.52 & 0.40 & 0.42 \\
\hline $\mathbf{G}_{\mathbf{1}}$ & 0.49 & 0.53 & 0.56 & 1.43 & 0.37 & 0.47 \\
\hline $\mathbf{G}_{\mathbf{2}}$ & 0.49 & 0.52 & 0.55 & 1.33 & 0.42 & 0.50 \\
\hline $\mathbf{G}_{3}$ & 0.39 & 0.51 & 0.55 & 1.43 & 0.40 & 0.46 \\
\hline $\mathbf{s}_{1} \mathbf{g}_{1}$ & 0.33 & 0.40 & 0.52 & 1.93 & 0.35 & 0.46 \\
\hline$s_{1} g_{2}$ & 0.45 & 0.44 & 0.46 & 1.74 & 0.42 & 0.46 \\
\hline $\mathbf{s}_{1} \mathbf{g}_{3}$ & 0.46 & 0.37 & 0.68 & 1.81 & 0.41 & 0.47 \\
\hline $\mathbf{s}_{2} \mathbf{g}_{1}$ & 0.61 & 0.34 & 0.54 & 1.49 & 0.38 & 0.48 \\
\hline $\mathbf{s}_{2} \mathbf{g}_{2}$ & 0.58 & 0.46 & 0.61 & 1.01 & 0.41 & 0.45 \\
\hline $\mathbf{s}_{2} \mathbf{g}_{3}$ & 0.45 & 0.60 & 0.58 & 1.01 & 0.45 & 0.45 \\
\hline $\mathbf{s}_{3} \mathbf{g}_{1}$ & 0.57 & 0.65 & 0.54 & 1.23 & 0.38 & 0.39 \\
\hline $\mathbf{S}_{3} \mathbf{g}_{2}$ & 0.54 & 0.46 & 0.60 & 1.28 & 0.38 & 0.52 \\
\hline $\mathrm{s}_{3} \mathrm{~g}_{3}$ & 0.34 & 0.46 & 0.39 & 1.52 & 0.39 & 0.39 \\
\hline $\mathbf{S}_{4} \mathbf{g}_{1}$ & 0.43 & 0.67 & 0.64 & 1.27 & 0.34 & 0.63 \\
\hline $\mathbf{S}_{4} \mathbf{g}_{2}$ & 0.48 & 0.64 & 0.35 & 1.04 & 0.45 & 0.58 \\
\hline $\mathbf{s}_{4} \mathbf{g}_{3}$ & 0.37 & 0.49 & 0.50 & 1.58 & 0.41 & 0.59 \\
\hline $\mathbf{S}_{5} \mathbf{g}_{1}$ & 0.53 & 0.60 & 0.55 & 1.71 & 0.40 & 0.41 \\
\hline $\mathrm{S}_{5} \mathrm{~g}_{2}$ & 0.39 & 0.59 & 0.73 & 1.61 & 0.45 & 0.49 \\
\hline $\mathbf{s}_{5} \mathbf{g}_{3}$ & 0.33 & 0.89 & 0.62 & 2.26 & 0.36 & 0.56 \\
\hline Treatment mean & 0.33 & 0.61 & 0.62 & 1.24 & 0.39 & 0.38 \\
\hline Control & 0.45 & 0.53 & 0.55 & 1.45 & 0.43 & 0.47 \\
\hline $\operatorname{SEm}( \pm): S$ & 0.48 & 0.92 & 0.54 & 1.69 & 0.65 & 0.40 \\
\hline G & 0.04 & 0.03 & 0.03 & 0.09 & 0.02 & 0.03 \\
\hline SG & 0.05 & 0.05 & 0.02 & 0.07 & 0.02 & 0.02 \\
\hline $\mathrm{CD}(0.05): \mathrm{S}$ & 0.083 & 0.057 & 0.051 & 0.156 & 0.024 & 0.009 \\
\hline G & NS & 0.076 & 0.086 & 0.267 & NS & 0.096 \\
\hline SG & NS & NS & NS & NS & NS & NS \\
\hline Treatment Vs Control & NS & 0.166 & 0.016 & 0.111 & 0.291 & NS \\
\hline
\end{tabular}


Table.4 Effect of nutrient sources, growth stages and their interaction on total soluble protein, $\mathrm{mg} \mathrm{g}^{-1}$

\begin{tabular}{|c|c|c|c|}
\hline Treatments & Booting & Flowering & Harvest \\
\hline \multicolumn{4}{|l|}{ Nutrient sources } \\
\hline $\mathrm{S}_{1}$ & 0.66 & 1.31 & 1.69 \\
\hline $\mathbf{S}_{\mathbf{2}}$ & 0.88 & 1.97 & 0.92 \\
\hline $\mathbf{S}_{3}$ & 1.04 & 1.84 & 0.98 \\
\hline $\mathbf{S}_{4}$ & 1.02 & 1.71 & 0.94 \\
\hline $\mathbf{S}_{5}$ & 0.70 & 1.89 & 1.03 \\
\hline \multicolumn{4}{|l|}{ Growth stages } \\
\hline $\mathbf{G}_{1}$ & 0.75 & 1.53 & 0.91 \\
\hline $\mathbf{G}_{\mathbf{2}}$ & 0.92 & 1.76 & 1.91 \\
\hline $\mathbf{G}_{3}$ & 0.90 & 1.95 & 1.51 \\
\hline \multicolumn{4}{|l|}{ Interaction effects } \\
\hline $\mathrm{s}_{1} \mathrm{~g}_{1}$ & 0.36 & 0.82 & 0.69 \\
\hline $\mathrm{s}_{1} \mathbf{g}_{2}$ & 0.92 & 1.59 & 0.74 \\
\hline $\mathbf{s}_{1} \mathbf{g}_{3}$ & 0.70 & 1.54 & 3.64 \\
\hline $\mathbf{s}_{2} \mathbf{g}_{1}$ & 0.82 & 1.58 & 0.62 \\
\hline $\mathbf{s}_{2} \mathbf{g}_{2}$ & 0.94 & 1.99 & 1.03 \\
\hline $\mathbf{s}_{2} \mathbf{g}_{3}$ & 0.89 & 2.33 & 1.11 \\
\hline $\mathbf{s}_{3} \mathbf{g}_{1}$ & 0.86 & 1.85 & 0.89 \\
\hline $\mathrm{s}_{3} \mathrm{~g}_{2}$ & 0.98 & 1.88 & 0.97 \\
\hline $\mathbf{s}_{3} \mathbf{g}_{3}$ & 1.27 & 1.78 & 1.09 \\
\hline $\mathrm{s}_{4} \mathrm{~g}_{1}$ & 0.95 & 1.43 & 0.95 \\
\hline $\mathbf{s}_{4} \mathbf{g}_{2}$ & 1.07 & 1.80 & 1.08 \\
\hline $\mathbf{s}_{4} g_{3}$ & 1.04 & 1.92 & 0.80 \\
\hline $\mathbf{s}_{5} \mathbf{g}_{1}$ & 1.77 & 1.98 & 1.39 \\
\hline $\mathbf{s}_{5} \mathbf{g}_{2}$ & 1.71 & 1.52 & 0.76 \\
\hline $\mathrm{s}_{5} \mathbf{g}_{3}$ & 0.61 & 2.18 & 0.94 \\
\hline Treatment mean & 0.85 & 1.74 & 1.11 \\
\hline Control & 0.703 & 1.78 & 0.74 \\
\hline $\operatorname{SEm}( \pm): S$ & 0.094 & 0.136 & 0.472 \\
\hline $\mathbf{G}$ & 0.072 & 0.205 & 0.366 \\
\hline SG & 0.161 & 0.232 & 0.770 \\
\hline $\mathrm{CD}(0.05): \mathrm{S}$ & 0.272 & 0.394 & NS \\
\hline G & NS & NS & NS \\
\hline SG & NS & NS & NS \\
\hline Treatment Vs Control & NS & NS & NS \\
\hline
\end{tabular}


Fig.1 Effect of nutrient sources on flag leaf area $\left(\mathrm{cm}^{2}\right)$ at different crop growth stages

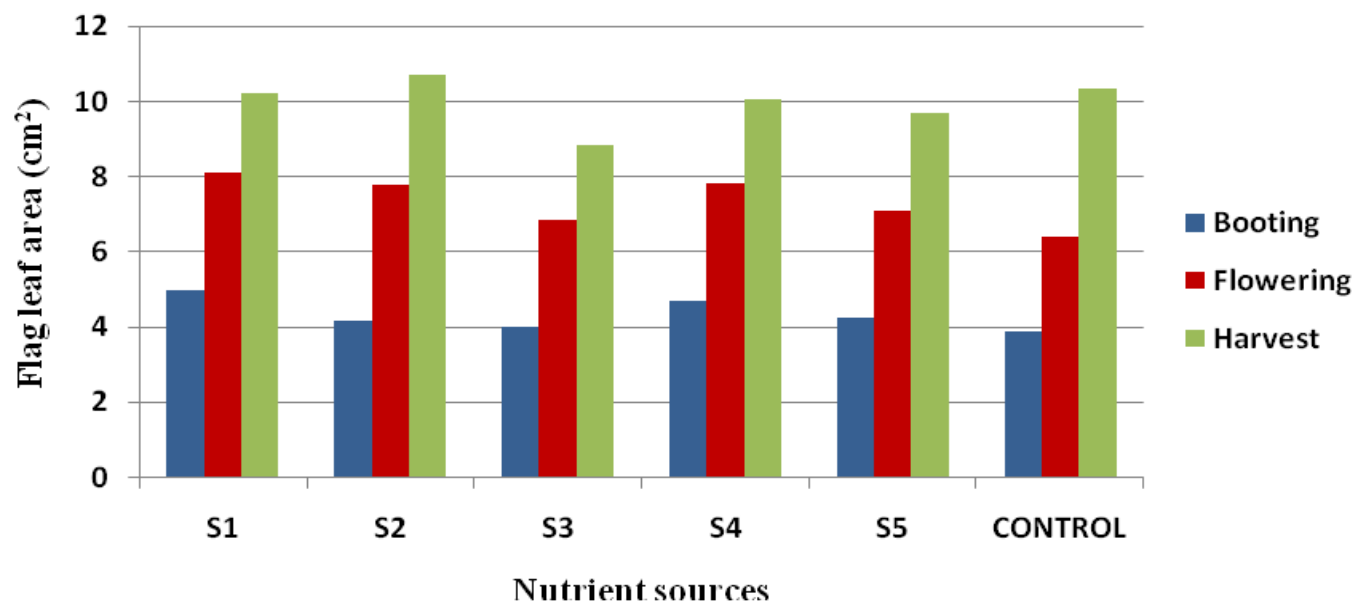

$S_{1}$ - Potassium nitrate, $S_{2}$ - Calcium nitrate, $S_{3}$ - Magnesium sulphate, $S_{4}-19: 19$ : 19 complex, $S_{5}-S_{2}+$ $\mathrm{S}_{3}+\mathrm{S}_{4}$, CONTROL - (KAU POP)

Fig.2 Effect of flag leaf nutrition with different nutrient sources on leaf area duration

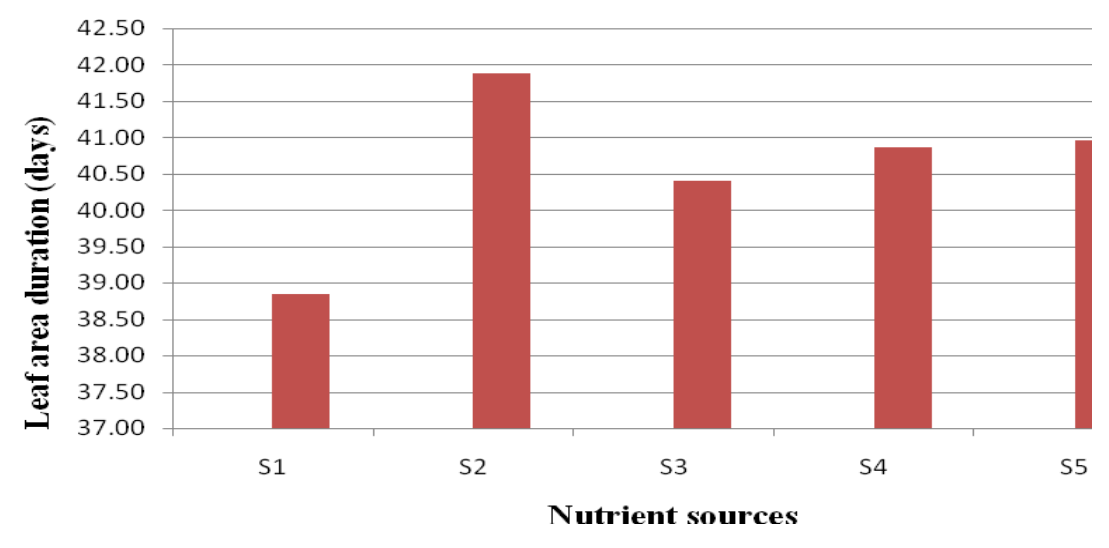

\section{Specific leaf weight}

Specific leaf weight (SLW) is a vital variable related to physiological processes occurring in plants. The present study revealed significant variation in SLW with nutrient sources and with the interaction between nutrient sources and crop growth stages (Table 1). SLW was higher $\left(2.11 \mathrm{~g} \mathrm{~m}^{-2}\right.$ ) with $\mathrm{S}_{5}$ (combination of calcium nitrate, magnesium sulphate and 19:19:19 complex) at flowering and with flag leaf nutrition of 19:19:19 complex @ 0.5 per cent at harvest $\left(2.11 \mathrm{~g} \mathrm{~m}^{-2}\right)$. In the case of interaction effect, application of potassium nitrate at booting stage recorded higher SLW. The better nutrient balance supported by the treatments might have contributed towards increasing the SLW. According to Braun and Wild (1984) and Field and Mooney (1986) specific leaf weight is very sensitive to plant nutrient status and nutrient application increases the specific leaf weight. Specific leaf weight, a measure of leaf thickness, has been reported to have a strong positive correlation with leaf photosynthesis of several crops as reported by Bowes et al., (1972). 
Dornhoff and Shibles (1970) presumed that higher SLW might be associated with higher cell surface to volume ratio and hence lower mesophyll resistance to $\mathrm{CO}_{2}$ entry and increase in photo as similates accumulation.

\section{Relative growth rate and net assimilation rate}

The nutrient sources exhibited significant effect on the relative growth rate and net assimilation rate (Table 1). FLN with potassium nitrate @ 0.5 per cent recorded maximum relative growth rate at flowering $\left(0.057 \mathrm{mg} \mathrm{g}^{-1}\right.$ day $\left.^{-1}\right)$ and harvest stages $(0.028$ $\mathrm{mg} \mathrm{g}^{-1}$ day $\left.^{-1}\right)$. Net assimilation rate recorded at booting ( $3.76 \mathrm{mg} \mathrm{cm}^{-2} \mathrm{day}^{-1}$ ) and flowering stages $\left(2.58 \mathrm{mg} \mathrm{cm}\right.$ day $\left.^{-1}\right)$ was also significantly superior with FLN of potassium nitrate @ 0.5 per cent. The improvement in RGR and NAR under the influence of potassium nitrate can be traced to the importance of potassium and nitrogen in improving the growth and photosynthesis of the crops. Kundu and Sarkar (2009) have highlighted the role of potassium in photosynthesis, by directly increasing growth and leaf area index and hence carbon dioxide assimilation enhances outward translocation of more ATP essential for vigorous growth of plants. The nitrogen supplied by potassium nitrate might have also contributed to higher RGR and NAR. Nitrogen, in general, due to its role in production and translocation of cytokinin from the root to the shoots might have increased cell division rate and growth rate of rice. Similar results have been reported by Timothy and Joe (2003). NAR represents plant photosynthetic efficiency. Higher net assimilation rate might be due to more dry matter production supported by FLN with potassium nitrate. The decrease in NAR at the later stages of growth could be attributed to an increase in the number of older leaves which lost photosynthetic activity (Pandey et al., 1978).
Plant pigments - chlorophyll and carotenoids

Chlorophyll content is of particular significance as an indicator of photosynthetic activity. The chlorophyll and carotenoid contents of leaf blade were significantly affected by the different nutrient sources at flowering stage (Tables 2 and 3). Highest chlorophyll content was recorded with FLN of 19:19:19 complex @ 0.5 per cent and highest carotenoid was with potassium nitrate @ 0.5 per cent. The chlorophyll and carotenoid contents were maximum for $\mathrm{S}_{5}$ (a combination of calcium nitrate, magnesium sulphate and 19:19:19 complex) in the flag leaf sheath. The foliar nutrition might have resulted in better photosynthetic rate resulting in more pigment formation with increased leaf area. Nitrogen concentration in green vegetation is related to chlorophyll content, and therefore indirectly to one of the basic plant physiological processes: photosynthesis (Bojovic and Stojanovic, 2005). Studies in rice (Tang, 2000) showed that nitrogen fertilizer application increased the chlorophyll content of leaves, photosynthetic rate of rice flag leaf, electron transport capacity of PS I and PS II and extended the duration of photosynthetic duration in leaves. The effect of the combination treatment on the carotenoid content could be attributed to the effect of magnesium in enhancing the carotenoid content as reported by Ding et al., (2008).

\section{Soluble protein}

Soluble substances like soluble proteins reflect the ability of plants in making osmotic adjustments. Magnesium sulphate@0.5 per cent $\left(S_{3}\right)$ recorded maximum total soluble protein content in flag leaf at booting (1.04 $\mathrm{mg} \mathrm{g}^{-1}$ ) and calcium nitrate @ 0.5 per cent $\left(\mathrm{S}_{2}\right)$ at flowering stage $\left(1.97 \mathrm{mg} \mathrm{g}^{-1}\right)$ (Table 4). High flag leaf soluble protein content 
might be due to better absorption of nutrients especially nitrogen, when applied as foliar nutrition. According to Minjun et al., (2002), the level of soluble protein is regarded as an important indicator of the degree of leaf senescence. The present study also revealed the role of soluble protein in delaying senescence of the flag leaf as evidenced by the higher leaf area duration recorded at harvest. The finding corroborate with those of Yan and Shi (2013).

The study revealed that supplementing the KAU POP recommendation for high yielding medium duration wetland rice (FYM @ 5 t $\mathrm{ha}^{-1}+90: 45: 45 \mathrm{~kg} \mathrm{NPK} \mathrm{ha}{ }^{-1}$ ) with flag leaf nutrition of potassium nitrate or 19: 19: 19 complex@0.5 per cent concentration, 5 days prior to booting and 50 per cent flowering stages resulted in significantly superior physiological response in lowland rice, measured in terms of leaf area duration, specific leaf weight, relative growth rate, net assimilation rate, plant pigments and soluble protein content.

\section{References}

Ashraf M, Akbar M and Salim M. 1994. Genetic improvement in physiological traits of rice yield. In: Slafer G.A., (ed.), Genetic Improvement of Field Crops. Marcel Dekker Incorporate, New York, pp. 413-455.

Bojovic, B. and Stojanovic, J. 2005. Chlorophyll and carotenoid content in wheat cultivars as a function of mineral nutrition. Arch. Biol. Sci. 57: 283-290.

Bowes G.W., Orgen, L., and Hageman R.H. 1972. Light saturated photosynthesis rate, $\mathrm{RuBp}$ carboxylase activity and specific leaf weight in soybean grown under different light intensities. Crop Sci. 12: 77-79.

Bradford, M.M. 1976. A rapid and sensitive method for the quantification of microgram quantities of protein utilizing the principles of protein dye binding. Anal. Biochem. 72: 248-254.

Braun, P. and Wild, M. 1984. The influence of brassinosteroid on growth and parameters of photosynthesis of wheat and mustard plants. J. Plant Physiol. 116: 189-196.

Bush, D.S. 1995. Calcium regulation in plant cell and its role in signaling. Аnnu. Rev. Plant Biol. 46: 95-122.

De Datta, S. K. 1981. Principles and Practices of Rice Production. John Wiley and Sons, New York, 618p.

Ding, Y.C., Chang, C.R., Lou, W., Wu, Y.S., Ren, X.L., Wang, P., and Xu, G.H. 2008. High potassium aggravates the oxidative stress induced by magnesium deficiency in rice leaves. Pedosphere 18 (3): 316-327.

Dornhoff, G.M. and Shibles, R.M. 1970. Varietal differences in net photosynthesis of soybean leaves. Crop Sci. 10: 42-45.

Fageria, N.K., Filho, M.P.B., Moreora, I.A., and Guimaraes, G.M. 2009. Foliar fertilization of crop plants. J. Plant Nutr. 32: 1044-1064.

Field, C. and Mooney, H.A. 1986. The photosynthesis-nitrogen relationship in wild plants. In: Givnich, T.V. (ed.), The Economy of Plant Form and Function. Cambridge University Press, New York, USA, pp. 25-55.

Kondratev, M.N., Konch, K.M.F., and Treatyakov, N.N. 1984. Role of Ca:K ration in soil, in uptake and assimilation of nitrate by maize. Investiya Timiryazav skoi sel Skohozya istrennot Akademi 3: 113-117.

Mackill, D. J., Coffman, W. R. and Garrity, D. P. 1996. Rainfed Lowland Rice Improvement. International Rice Research Institute, Manila, Philippines, $76 \mathrm{p}$.

Minjun, X., Guiru, L., Xueju, Y., and Lijun, 
W. 2002. The influence of water stress to protein metabolism of winter wheat cultivars with different drought resistance ability. Agric. Res. Arid Areas 20(3): 85-88.

Misra,A.N., Sahu,S., Misra,M., Mohapatra,P., Meera, I. and Das, N. 1997. Sodium chloride induced changes in leaf growth and pigment and protein contents in two rice cultivars. Biologia Plantarum 39: 257-262.

Pandey, R. K., Saxene, M. C., and Singh, V. B. 1978. Effect of moisture stress on growth, yield and yield component of field grown sorghum variety having glossy and non-glossy leaf. Indian $J$. Agric. Sci. 53: 428-430.

Pearce, R.B., Brown, R.H., and Balster, R.F. 1968. Photosynthesis of alfalfa leaves as influenced by age and environment. Crop Sci. 6: 677-680.

Tambussi EA, Bort J, Guiamet JJ, Nogués S and Araus JL. 2007. The photosynthetic role of ears in $\mathrm{C} 3$ cereals: metabolism, water use efficiency and contribution to grain yield. Crit. Rev. Plant Sci.26: 116.

Tang, X.R. 2000. Effect of N supply on yield and on protein content and its mechanism in hybrid rice. Hybrid Rice 15 (2): 34-37.

Tari, D. B., Gazanchian, A., Perdashti, H. A. and Nasiri, M. 2009. Flag leaf morphophysiological response to different agronomical treatments in a promising line of rice (Oryza sativa L.). American-Eurasian J. Agric. Environ. Sci. 5: 403-408.

Timothy, W. and Joe, E. 2003. Rice Fertilization. Information sheet No. 1341, Mississippi Agricultural and Forestry Experiment Station, USA, 4p.

Watson, D.J. 1947. Comparative physiological studies on the growth of field crops: variation in net assimilation rate and leaf area between species and varieties and within and between years. Ann. Bot. 11: 47-76.

Williams, R.F. 1946. The phenology of plant growth with special reference to the concepts of net assimilation rate. Ann. Bot. 10: 41-72.

Yan, L. and Shi, Y. 2013. Effect of nitrogen application rate on the enzyme activity of flag leaf after anthesis. Adv. J. Food Sci. Technol. 5: 738-742.

Yang, J.C., Zhang,J.H., Wang,Z.Q.and Zhu, Q. S. 2003. Hormones in the grains in relation to sink strength and post anthesis development of spikelets in rice. Plant Growth Regulators 41:185195.

Yoshida, S., Forno, D.A., Cock, J.H., and Gomez, K.A. 1976. Laboratory Manual for Physiological Studies of Rice (3rd Ed.). International Rice Research Institute, Los Banos, Philippines, 83p.

\section{How to cite this article:}

Shalini Pillai, P. and Surya, M. S. 2021. Physiological Response of Flag Leaf Nutrition in Rice (Oryza sativa L.). Int.J.Curr.Microbiol.App.Sci. 10(02): 1852-1862. doi: https://doi.org/10.20546/ijcmas.2021.1002.220 